\title{
Macrophage migration inhibitory factor inhibits BMP-4 induced apoptosis
}

\author{
Nicholas Matluk $^{1,2}$, Tamara Adams ${ }^{1}$, Aldona Karaczyn ${ }^{1}$, Joseph Verdi $^{1 *}$ \\ ${ }^{1}$ Maine Medical Center Research Institute, Research Drive, Scarborough, USA \\ ${ }^{2}$ Molecular and Biomedical Sciences, Hitchner Hall, Orono, USA \\ Email: verdij@mmc.org
}

Received 5 September 2012; revised 11 October 2012; accepted 4 November 2012

\begin{abstract}
Non-canonical bone morphogenic protein (BMP) pathway signaling plays a critical role during embryonic neurogenesis, inducing apoptosis and eliminating an excess of neural progenitor cells, in preparation for the population of the neural stem cell niches. Our previous work discovered that non-canonical BMP signaling also induced the expression of the antiapoptotic cytokine macrophage migration inhibitory factor (MIF). Because there are residual neural progenitors that escape BMP induced apoptosis, we believed the expression of MIF could be used to counter this specific type of developmental apoptosis. In vitro studies using P19 cells and ex vivo studies using E13 neural progenitor from wild type and mif-/- mice revealed that overexpression of mif was able to counteract BMP induced apoptosis, while in contrast ablation of mif resulted in a dramatic increase in apoptosis. Despite this finding, it is interesting to note that mif-/- mice, while presenting an overall increase in apoptosis are viable, ablation of mif expression in zebrafish and frog are embryonic lethal, suggesting a redundant or non-critical role of MIF in the developing mammalian embryo.
\end{abstract}

Keywords: MIF; BMP; Apoptosis; Embryonic; Neural Stem Cell

\section{INTRODUCTION}

\section{BMP Pathway}

The formation of the neural stem cell niches and differentiation of neural progenitor cells require a complex balance of precisely timed intrinsic and extrinsic signals during development. During murine neurogenesis, activation of the bone morphogenic protein (BMP) pathway is one specific signal. The BMP pathway is a critical signal transduction pathway that plays a role in prolifera-

\footnotetext{
"Corresponding author.
}

tion, differentiation, cell cycle, apoptosis, and embryogenesis [1]. Two distinct pathways, the canonical or SMAD dependent and the non-canonical Tak1 dependent pathway mediate signal transduction of BMP [2]. At the onset of corticogenesis, neural progenitors in E13.5 mice undergo Tak1/p38MAPK dependent induced apoptosis when stimulated with BMP [3-5]. By E16, the middle of corticogenesis, BMP induces the differentiation and proliferation of those stem cells that survived the earlier culling. Our latest paper [6], showed that upon BMP stimulation, the expression of the anti-apoptotic cytokine macrophage migration inhibitory factor (MIF) was dramatically increased in both 293HEK cells and P19 cells via a NF-кB dependent mechanism. Therefore we though it prudent to evaluate the role of MIF in BMP induced apoptosis.

MIF is constitutively and ubiquitously expressed throughout mammalian embryogenesis, organogenesis and adulthood [5-10], and is not unique to humans or mice, but is found to be highly conserved across various species, including rats, chickens, malaria, zebrafish, cow, wild boar, sheep, salmon, frog, rhesus monkey, corn, dog, silkworm, chimpanzee, and pufferfish.

Unlike other cytokines, MIF is unique in that it has multiple receptor partners and can be endocytosed. The major cognate receptor for MIF is CD74 also known as the major histocompatibility complex class II invariant chain [10]. CD74 contains a short intracellular sequence that cannot interact with signal transduction molecules, therefore it heterodimerizes with CD44 [11,12], CXCR2 [13] or CXCR4 [13-16] to propagate its signal, where it regulates cellular invasion, migration, and proliferation.

The endocytosis of MIF is controlled by $\beta$-arrestin1 through CD74/CD44 dependent and independent mechanisms [17]. Intracellular MIF can increase proliferation through activation of the JNK/AP1 pathway and degradation of p27 [18]. Intracellular MIF can also directly bind to and inhibit the phosphorylation of p53 [19]. This inhibitory effect of MIF on p53 has been shown to inhibits nitric oxide induced apoptosis caused by macrophage 
secretion [19] regulate DNA damage [20,21], accelerates the repair of nerve fibers by inhibiting Schwann cell induced apoptosis [22,23], impair the inflammatory response of arthritis [24-26], and blocks the tumor suppressor functions of p53 [7-10]. Knockout models of MIF show variable levels of p53 function. Ablation of MIF expression in the zebrafish by morpholino oligonucleotide resulted in a $40 \%$ lethality rate and an increase in apoptosis in the embryos that did survive [11,12]. Ablation of MIF expression in the frog by morpholino was $100 \%$ lethal, as the affected embryos were devoid of neural tissue, notochord, and neural tube [13]. In contrast, the MIF knockout mouse model while healthy and viable has impaired DNA checkpoint damage caused by an increase in p53 activity.

To determine the role of MIF in regulating the noncanonical BMP pathway we stimulated the P19 mouse embryonic carcinoma cell line with BMP-4 and retinoic acid to induce apoptosis. It was found that overexpression of a MIF:EGFP protein or stimulation of cells with rMIF decreased both the amount of phospho-p53 and the number of Annexin V and 7AAD double positive apoptotic cells. In contrast, apoptosis was increased in P19 cells transfected with a MIF shRNA vector. This effect was duplicated in E13.5 neural progenitors isolated from wild type and mif-/- embryos. Overall, the studies herein provide novel insight into how neural progenitors escape BMP-4 induced apoptosis leaving behind a small subset of stem cells.

\section{RESULTS}

\subsection{MIF Counteracts BMP-4 Apoptosis}

Building on previous findings that non-canonical BMP signaling induces apoptosis through phosphorylated p53, and that the expression of the anti-apoptotic cytokine MIF [6] is linked to BMP signaling, we sought to determine if MIF could counteract BMP-4 induced apoptosis, since MIF can prevent the phosphorylation of p53 [7]. We used the P19 cell line; a mouse embryonic teratocarcinoma cell line derived from the $\mathrm{C} 3 \mathrm{H} / \mathrm{He}$ mouse strain and has been used extensively for BMP pathway signaling and neural apoptosis [5,14-16].

A P19 cell line stably transfected with MIF:EGFP fusion protein showed a decrease in phosphorylated p53 at 24 and $48 \mathrm{hr}$ post BMP stimulation when compared to a GFP control cell line. In contrast, a P19 cell line stably transfected with a MIF shRNA plasmid showed an increase in phosphorylated p53 at 24 and 48 hr post BMP stimulation when compared to a GFP control cell line (Figure 1(a)). Manipulation of mif expression did not change total p53 concentration (Figures 1(a) and (b)). To confirm that these results were not a function of transfection and stable line creation, we stimulated wild-type P19

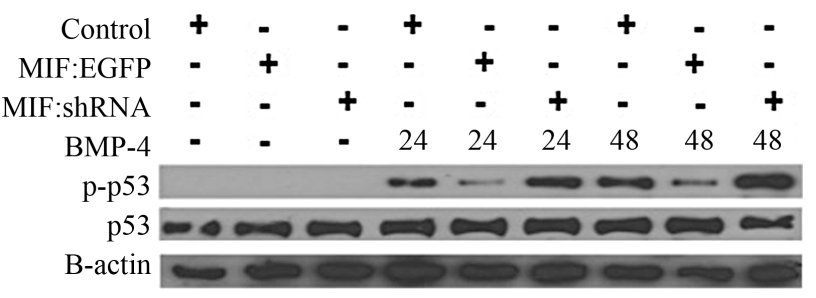

(a)

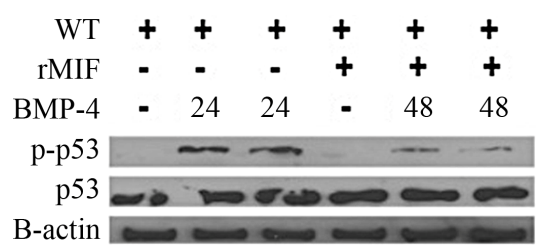

(b)

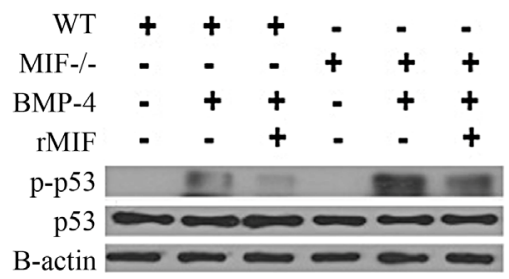

(c)

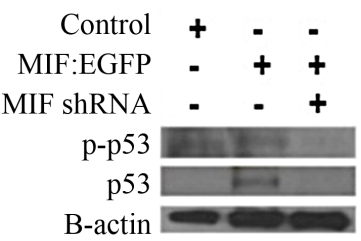

(d)

Figure 1. MIF counteracts BMP-4/RA induced phospho-p53. (a) Analysis of phospho-p53 was used to determine the amount of apoptosis in vector control, MIF:EGFP and MIF shRNA P19 stable cell lines after $10 \mathrm{ng} / \mathrm{ml} \mathrm{BMP-4}$ and $1 \mathrm{uM}$ retinoic acid treatment for 48 hours; (b) Analysis of phospho-p53 was used to determine the amount of apoptosis in wild-type P19 incubated with $50 \mathrm{ng} / \mathrm{mL}$ rMIF cells after $10 \mathrm{ng} / \mathrm{ml}$ BMP-4 and $1 \mathrm{uM}$ retinoic acid treatment for 48 hours; (c) Analysis of phosphop53 was used to determine the amount of apoptosis in E13.5 neural progenitors from mif-/- mice and wild-type mice when stimulated with BMP-4 and with or without rMIF; (d) Western blot showing the expression of MIF in vector control, MIF:EGFP, and MIF shRNA P19 cell lines.

cells with $50 \mathrm{ng} / \mathrm{ml}$ of rMIF in the presence BMP-4 and retinoic acid. It was found that the addition of rMIF to the media was able to substantially reduce the amount of phosphorylated p53 (Figure 1(b)). Figure 1(d) shows the protein expression of MIF in undifferentiated P19 cells, stably expressing either a MIF: EGFP fusion protein or MIF shRNA. P19 cells expressing a control scrambled shRNA were found to endogenously express MIF, albeit at a low level. Stable transfection with a MIF: EGFP fusion protein did not increase the amount of endogenous MIF, while the MIF shRNA completely ablated MIF expression. 
MIF is ubiquitously expressed during embryogenesis $[11,13,17,27]$ and a vast majority of, but not all cortical neural progenitors cells undergo BMP induced apoptosis starting on day 11 , culminating on day 13.5 [3,5]. Therefore, to test if MIF regulates apoptosis ex vivo, we added $50 \mathrm{ng} / \mathrm{ml}$ rMIF to cultures of cortical neural progenitors cells isolated from wild-type and mif-/- mice treated with BMP-4 (Figure 1(c)). In comparison to wild type progenitors, mif-/- progenitor cells had a pronounced increase in phosphorylated p53 after BMP stimulation. Similar to the results obtained when using P19 cells, the addition of rMIF to ex vivo neural progenitor cells was able to reduce BMP-4 induced phosphorylation of p53 in both wild type and mif-/- cells.

To further illustrate that MIF expression regulates BMP induced apoptosis and not just the phosphorylation status of p53, we performed Annexin-V staining to analyze the percentage of apoptotic cells after BMP-4 and retinoic acid treatment in P19 cells (Figure 2(a)). Figure 2(b) compares the percentage of Annexin V+/7AAD + cells in each sample. The addition of recombinant MIF was able to influence the amount of Annexin V+ significantly in the as the percentage of Annexin V+ cells was reduced in the vector control cells from $65.63 \% \pm 0.82 \%$ without rMIF to $35.38 \% \pm 0.45 \%$ with rMIF (p-value of $6.77 \times 10^{-8}$ ). This effect was also seen in the MIF shRNA cells, in which percentage of Annexin $\mathrm{V}+$ cells was reduced from $97.03 \% \pm 0.21 \%$ without endogenous or rMIF to $37.40 \% \pm 1.24 \%$ with rMIF (p-value of $1.47 \times$ $\left.10^{-8}\right)$. Comparison of the MIF shRNA cell line $(97.03 \%$ $\pm 0.21 \%$ apoptotic cells) to the control cells $(65.63 \% \pm$ $0.82 \%$ apoptotic cells) also suggests that endogenous and BMP induced MIF can act as antiapoptotic autocrine factor in tempering BMP induced apoptosis (p-value of $\left.3.91 \times 10^{-8}\right)$. Finally, after BMP/-RA treatment, the MIF:EGFP stable line only contained $10.12 \% \pm 0.23 \%$ Annexin V+ cells, suggesting that constitutively high expression of MIF can have an inhibitory or protective effect against BMP-4/RA induced apoptosis, with a pvalue of $4.10 \times 10^{-9}$ when compared to control cells stimulated with BMP-4 and retinoic acid. The slightly elevated levels of PE staining seen even in the control samples is due to the trypsinization of the P19 cells.

\subsection{MIF Expression Does Not Affect Retinoic Acid Induced Neuronal Differentiation}

The P19 mouse embryonic carcinoma cell line has been used extensively for neural differentiation experiments as these cells share many properties in common with neural progenitors [18]; Kendall SE, 2005 \#1\}. We differentiated the MIF: EGFP, MIF shRNA and vector control cell lines using retinoic acid to determine if MIF can keep P19 cells in a progenitor cell state. RT-PCR and ICC

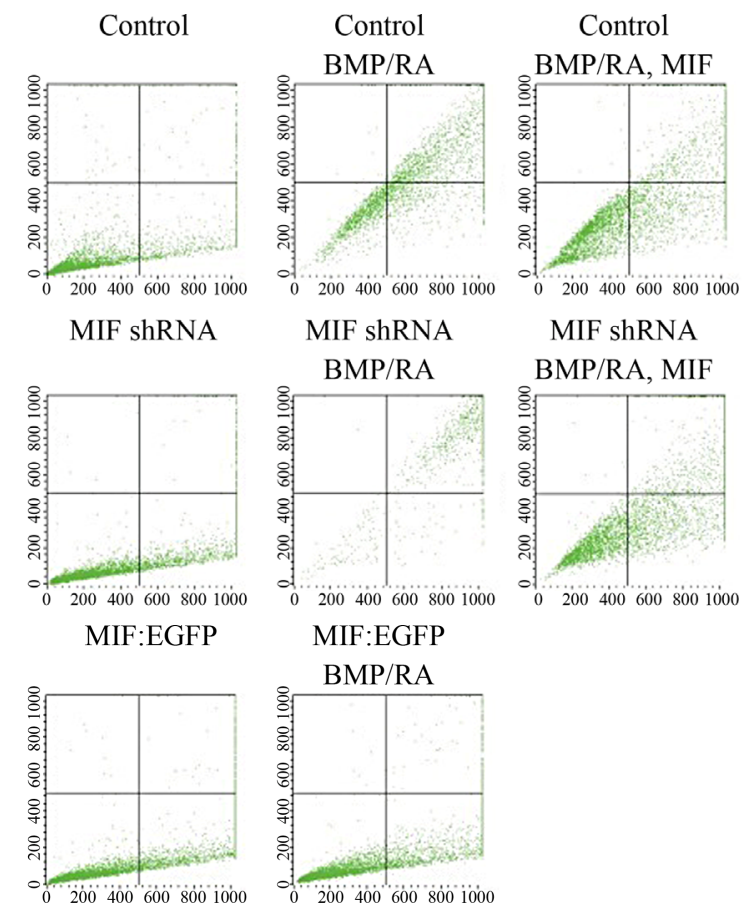

(a)

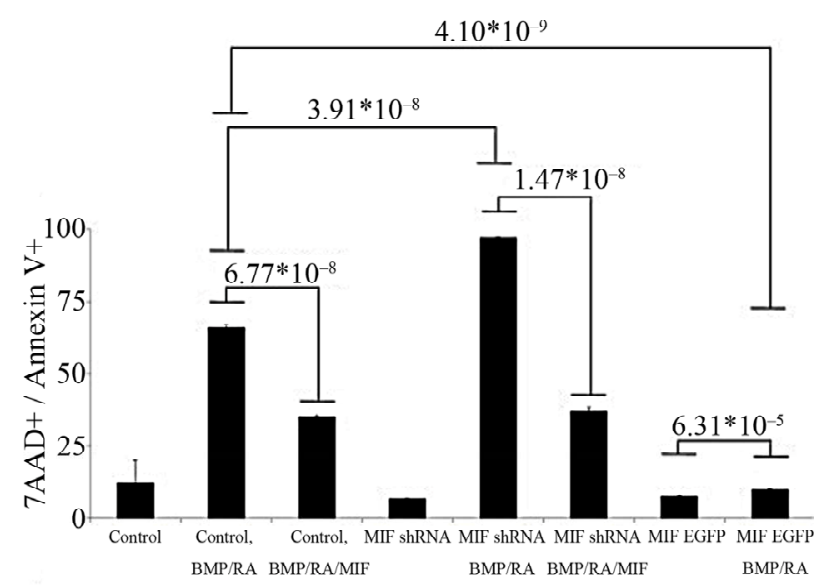

(b)

Figure 2. MIF counteracts BMP-4/RA induced apoptosis in P19 cells. Annexin-V and 7AAD labeling was used to determine the amount of apoptosis in vector control, MIF:EGFP and MIF shRNA P19 stable cell lines after $10 \mathrm{ng} / \mathrm{ml} \mathrm{BMP-4}$ and $1 \mathrm{uM}$ retinoic acid treatment for 48 hours. The addition of rMIF was used to rescue the cells from apoptosis. Data are representative of a single experiment performed in triplicate. p-values were determined by the two-tailed unpaired Student's $t$ test.

were used to analyze pluripotent stem cell transcription factor expression and differentiation markers (Figure 3). The data show that the expression of Nanog, Sox2 and Oct-4 are repressed after differentiation independent of MIF expression (Figure 3(a)). It was also found that upon differentiation, neurons (MAP2a/b and $\beta$-III tubulin), and glia (GFAP, MBP, S-100) were formed, also independent of MIF expression (Figure 3(a)). ICC was used to 


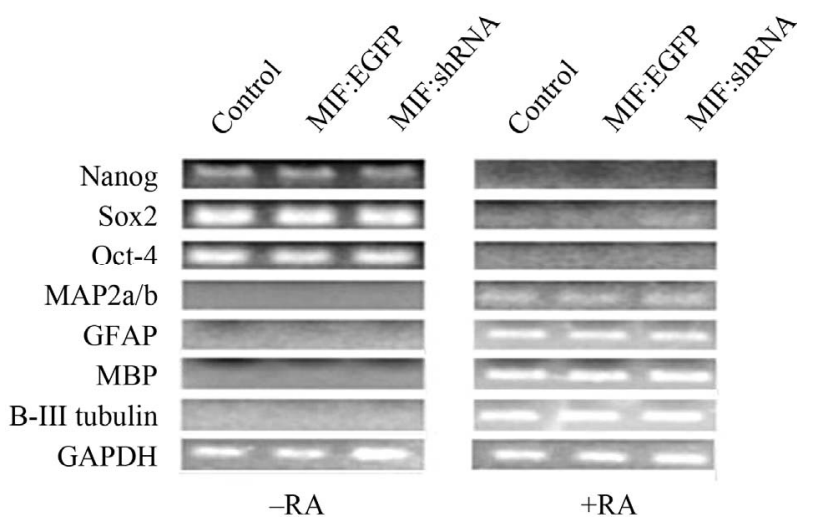

(a)

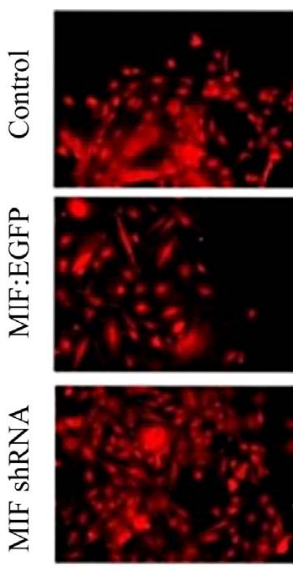

S-100

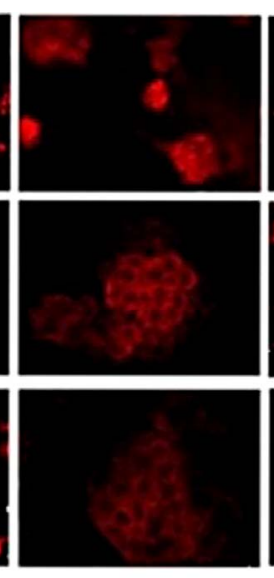

MBP
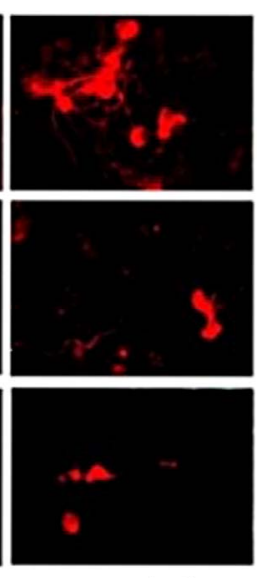

B-III tubulin

(b)

Figure 3. MIF expression does not influence retinoic acid induced differentiation. P19 cells stably transfected with a vector control, MIF:EGFP fusion protein, or MIF shRNA were differentiated via $1 \mathrm{uM}$ retinoic acid. (a) RT-PCR of stem cell transcription factors and markers for neurogenesis and glialgenesis before and after retinoic acid induced differentiation; (b) Immunofluorescence of neurogenesis and glialgenesis markers after retinoic acid induced differentiation.

visualize the phenotypic architecture of the differentiated cells (Figure 3(b)). Because there was no change in between stemness markers and neural and glial markers, it can be concluded that MIF expression does not influence retinoic acid induced differentiation.

\section{DISCUSSION}

\section{The Role of MIF during Neural Development}

While our research shows that MIF inhibits BMP-4 induced apoptosis, in vitro and ex vivo, it does not begin to fully address why the mouse knockout model is healthy and viable [19] when the zebrafish [11] and frog [13] knockdown models are embryonic lethal. Zebrafish mutants have malformed fins, jaws, retina, decreased neural tissue, and an increase in apoptosis as depicted by pyknotic nuclei and acridine orange staining. Frog mutants are

completely devoid of neural tissue, notochord, and neural tube. This is in dramatic contrast to the mif-/- mouse which only has variable levels if p53 activity, and no known development neural defects suggesting a noncritical or redundant role of MIF during development. Our finding that retinoic acid directed neural differentiation of P19 cells proceeds normally independently of MIF expression is very different then the findings of embryonic neural differentiation in the zebrafish and the frog. The increase in apoptosis seen in these models could eliminate the neural stem cell niche prior to neural differentiation or cause migrating neural crest cells to undergo apoptosis before populating the neural stem cell niche. The previous research on zebrafish and frog mif mutants did not study neural crest and neural stem cells, and therefore warrants further exploration. Another reason for the disparity between animal models could be cellular reduction-oxidation pathways.

Redox pathways play an important role before, during, and after implantation of the mammalian embryo. Prior to the development of uteroplacental circulation anoxic and hypoxic environments surround the developing embryo. Protection of the embryo from oxygen related stress is partially regulated by thioredoxin [20] and glutathione [21], GPX4, G6PD, selenoprotein W, and lipoic acid [22]. Disruption in redox protein activity during the early stages of development can result in build-up of reactive oxygen species (ROS) caused by normal metabolism [27], teratogenic effects caused by the maternal intake of chemicals, such as phenytoin, ethanol, nicotine, and thalidomide [23], or maternal diabetes [22]. Proper development of the embryo depends upon the relative redox state of the embryo. Disruption of classical redox protein expression can be fatal. For example, thioredoxin null mice die at E7.5, G6PD deficient dams have an increase in birth defects, and GPX4 deficient mice die mid-gestation through a lack of structural compartmentalization [22]. In comparison, the addition of thioredoxin to mouse embryo culture results in a greater probability of survival [23].

Macrophage migration inhibitory factor (MIF) is also a known redox protein containing the catalytic thiolprotein CXXC motif $[24,25]$ and studies have shown it functions as such in myocardial ischemia [26], nerve protection [28] and protein chaperoning [25]. During pregnancy, MIF protein can be found in the oviduct, uterus and amniotic fluid and MIF mRNA can be found in ovulated oocytes, zygotes, 2-cell embryos, 8-cell embryos, and blastocysts [17,29-31]. There is also nuclear staining of MIF protein in the extravillious trophoblast and cytotrophoblast and upregulation of MIF on gestation day 10.5 in the placenta [32]. In fact, Suzuki et al. [17] suggested in 1996 that MIF may act as a redox protein during embryogenesis. The development of both 
frog and zebrafish embryos is ex utero and independent of a maternal "host" to bathe the embryo in secreted factors, suggesting that the loss of a single potential redox protein is fatal. In contrast, the development of a mammalian embryo in utero is subjected to the expression of secreted maternal proteins that may counter the loss of non-critical redox protein such as MIF.

\section{MATERIALS AND METHODS}

\subsection{Cell Culture}

Wild-type P19 cells were cultured in $\alpha$ MEM media supplemented with $10 \% \mathrm{FBS}$, and gentamycin at $37^{\circ} \mathrm{C} 5 \%$ $\mathrm{CO}_{2}$. Cells were trypsinized and passaged at $75 \%$ confluency. Transfection was performed using Genejuice (EMDBiosciences, Darmstadt, Germany) as per the manufacturers' instructions for the creation of stable lines. Full length MIF (Origene, Rockville, MD, Cat. sc118641) was cloned into the mammalian expression vector pEGFPN3 (Clonetech, Mountain View, CA, Cat. PT3052-5). The pEGFP-N3 vector codes for a neomycin resistance gene and was used to aid in the creation of a MIF:EGFP stable line. The MIF shRNA (Origene Rockville, MD, Cat. TG319111) stable and control shRNA lines were created by limited-dilution sub-cloning. All cells were first serum starved for 4 hours prior to stimulation with either PBS or DMSO, varying concentrations of rMIF (R\&D Systems, Minneapolis, MN, Cat. 1978-MF/CF).

\subsection{Western Blotting}

Cells were serum starved for $\sim 4$ hrs prior to the addition of $10 \mathrm{ng} / \mathrm{ml}$ of BMP-4 (R\&D systems, Minneapolis, MN) or cell were transfected with respective vectors for $24 \mathrm{hrs}$ prior to being lysed with either RIPA buffer supplemented with tyrosine phosphatase inhibitors (Upstate, Billerica, MA Cat. 20-203), Ser/Thr phosphatase inhibittors (Upstate Cat. 20-204) and Protease Inhibitor Cocktail Set III (Calbiochem, Darmstadt, Germany Cat. 539134). This cocktail includes $100 \mathrm{mM}$ AEBSF (serine protease), $80 \mu \mathrm{M}$ Aprotinin (serine proteases), $4.5 \mathrm{mM}$ Bestatin (aminopeptidase B and leucine aminopeptidase), $1.5 \mathrm{mM}$ E-64 (cysteine proteases), $6.2 \mathrm{mM}$ Leupeptin Hemisulfate (cysteine proteases and trypsin-like Proteases), and 1 $\mathrm{mM}$ Pepstatin A (aspartic proteases). Lysates were then centrifuged at 10,000 rpm and the supernatant stored at $-80^{\circ} \mathrm{C}$ until use. Protein concentration was measured via Pierce BCA Kit (Rockford, IL, Cat. 23235) per manufacturer's instructions.

For the detection of protein expression by western blot, cells lysates were boiled for 5 min in $5 \times$ SDS-Page loading buffer, and then loaded into the wells of a $10 \%$ SDSPage gel, electrophoresed for $15 \mathrm{~min}$ at $60 \mathrm{~V}$, then for 90 min at $110 \mathrm{~V}$ on a Bio-Rad Power Pac electrophoresis system. The gel was then transferred to nitrocellulose membrane (Amersham Biosciences) for 2 hrs at $100 \mathrm{~mA}$ on ice. The nitrocellulose was then blocked with $4 \%$ non-fat dry milk in TBST for 1hour at RT. For the detection of phospho-specific proteins, 4\% BSA was used instead of non-fat dry milk. Primary antibodies diluted in blocking buffer were added to the nitrocellulose for overnight incubation at $4^{\circ} \mathrm{C}$ with agitation. The nitrocellulose was then washed three times with TBST, incubated with secondary antibody diluted in blocking buffer for 1 hour at RT. The membrane was then washed again with TBST, and enhanced chemiluminescent substrate (Denville, Cat. E2400) was sprayed onto the membrane and allowed to incubate for $1 \mathrm{~min}$. Excess reagent was removed and the nitrocellulose wrapped in plastic and developed in a dark room using Sensitive Blue X-ray film (Pegasus Scientific).

The following antibodies were used for western blotting: mouse anti $\beta$-actin (Novus Littleton, CO, Cat. NB 600-501) (1:10000 dilution), rabbit anti GFP (Santa Cruz, Cat. sc-8334) (1:1000 dilution), and rabbit anti MIF (Santa Cruz, Cat. sc-20121) (1:1000 dilution), mouse anti p53 (Abcam, Cambridge, MA, Cat. ab76242) (1:500 dilution rabbit anti phospho-S46 p53 (Abcam, Cambridge, MA, Cat. ab76242) (1:500 dilution). The following secondary antibodies were all diluted to 1:3000; goat anti rabbit HRP (Bio-Rad, Hercules, CA, Cat. 170-6515), goat anti mouse HRP (Bio-Rad, Cat. 170-6516).

\subsection{Isolation of Cortical Progenitor Cells}

Isolation of cortical progenitor cells was accomplished by using methods previously described [3]. Timed pregnant wild-type C57/B6 mice and mif-/- mice were euthanized at E13, embryos removed, and placed in Hank's balanced salt solution (HBSS) supplemented with $10 \%$ FBS. The brain was removed, freed of meninges and remaining cells were gently minced, briefly trypsinized with $0.05 \%$ trypsin-EDTA, and strained through a 1 micron cell strainer to disperse cells. Cells were plated on polyD-lysine coated cell culture wells at a concentration of 2 $\times 10^{5}$ cells/well in 60\% DMEM:F12, 38\% Neurobasal medium, 2\% FBS, and supplemented with N2 media and penicillin-streptomycin. Cells were allowed to adhere overnight, prior to apoptosis induction.

\subsection{Induction of Apoptosis}

BMP-4 and retinoic acid induced apoptosis in wild-type, vector control, MIF:EGFP, and MIF shRNA P19 cells was accomplished by using methods previously described [3]. Cells were plated on bacteriological grade culture dishes at a concentration of $1 \times 10^{5}$ cells/plate in alpha-MEM, 1\% FBS. Apoptosis was induced by incubation with $1 \mathrm{uM}$ all trans-retinoic acid and $10 \mathrm{ng} / \mathrm{ml}$ BMP- 
4 for 48 hours. Cortical progenitor cells were stimulated with the same concentration of retinoic acid and BMP-4 on poly-D-lysine coated cell culture wells. Analysis of apoptosis was analyzed two ways. Cell lysates were collected for analysis of total and phospho-p53 status by western blotting. The detection of apoptotic cells was accomplished using the Annexin V-PE Apoptosis Detection Kit I (BD Pharmingen, San Diego, CA, Cat. 5597). Following the manufactures instructions, stimulated cells were washed twice with cold PBS and resuspended in 1 $\mathrm{ml}$ of $1 \times$ binding buffer. One hundred microliters of resuspend cells were transferred to a new tube and $5 \mu$ of $\mathrm{PE}$ Annexin V and $5 \mu \mathrm{l}$ 7-AAD were added. Cells were gently mixed and allowed to incubate in the dark at room temperature for $15 \mathrm{~min}$. Four hundred microliters of $1 \times$ binding buffer was added to each tube and the cells analyzed by flow cytometry.

\subsection{Differentiation of P19 Cells}

Neural differentiation of P19 cells was accomplished by using retinoic acid as previously described [3]. Cells were grown in aggregates on sterile bacterial grade culture dishes in the presence of $1 \mathrm{uM}$ retinoic acid for four days. Cells were then trypsinized, transferred to fibronectin-coated tissue culture plates and cultured for an additional four days without the presence of retinoic acid. RNA was isolated using the RNeasy mini kit (Qiagen, Valencia, CA, Cat. 74104) and cDNA was created using Superscript II (Invitrogen, Carlsbad, CA, Cat. 18064). The following primers used for the analysis of retinoic acid induced neural and glial differentiation of P19 cells; Nanog forward primer:

5'-AGGGTCTGCTACTGAGATGCTCTG-3', Nanog reverse primer:

5'-CAACCACTGGTTTTTCTGCCACCG-3', Sox2 forward primer: 5'-AAAGCGTTAATTTGGATGGG-3', Sox 2reverse primer:

5'-ACAAGAGAATTGGGAGGGGT-3', Oct4 forward primer: 5'-TCTTCTGCTTCAGCAGCTTG-3', Oct4 reverse primer: 5'-GTTGGAGAAGGTGGAACCAA-3', Map2a forward primer:

5'-GCTGGTGGTATGTTCTGGCT-3', Map2a reverse primer: 5'-TACCGGTTCCTCAGCTTGTC-3', GFAP forward primer: 5'-TTTCTCGGATCTGGAGGTTG-3', GFAP reverse primer:

5'-AGATCGCCACCTACAGGAAA-3', MBP forward primer: 5'-TCTGCTGTGTGCTTGGAGTC-3', MBP reverse primer: 5'-GGCCTCAGAGGACAGTGATG-3', BIII-tubulin forward primer:

5'-GCAGGTCTGAGTCCCCTACA-3', BIII-tubulin reverse primer: 5'-CAGTGCGGCAACCAGATAG-3', GAPDH forward primer:

5'-TGGTCTACATGTTCCAGTATG-3', GAPDH reverse primer: 5'-TCCACCACCCTGTTGCTGTA-3'.

\subsection{Immunohistochemistry}

Immunofluorescence was performed as previously described [14]. The following primary antibodies were used: S-100 (Sigma, St. Louis, MO, Cat. S-2644), MBP (Boehringer, Ingelheim am Rhein, Germany Cat. 1118099), $\beta$ III-tubulin (Stem Cell Technologies, Vancouver, BC, 01409). The following secondary antibodies were used: goat anti mouse Alexa fluor 488 (Molecular Probes Carlsbad, CA, Cat. A-11029) (1:100) or goat anti rabbit Alexa fluor 488 (Molecular Probes, Cat. A-11008) (1:100) in blocking buffer goat anti mouse Alexa fluor 546 (Molecular Probes Carlsbad, CA, Cat. A-11030) (1:100) or goat anti rabbit Alexa fluor 546 (Molecular Probes, Cat. A-11010) (1:100). The cells were then washed with PBST, counterstained with DAPI and stored at $4^{\circ} \mathrm{C}$. Images were taken on a Zeiss Axiovert 200 fluorescent microscope and pseudo colored with MetaMorph software version 6.1 (Universal Imaging Corporation, Sunnyvale, CA). Co-localization is depicted by yellow pseudo-coloring in images using Alexa 488 and Alexa 546.

\section{REFERENCES}

[1] Streit, A. and Stern, C.D. (1999) Neural induction. A bird's eye view. Trends in Genetics, 15, 20-24. doi:10.1016/S0168-9525(98)01620-5

[2] Wilson, N.H. and Stoeckli, E.T. (2012) Sonic Hedgehog regulates Wnt activity during neural circuit formation. Vitamins \& Hormones, 88, 173-209. doi:10.1016/B978-0-12-394622-5.00008-0

[3] Kendall, S.E., Battelli, C., Irwin, S., Mitchell, J.G., Glackin, C.A. and Verdi, J.M. (2005) NRAGE mediates p38 activation and neural progenitor apoptosis via the bone morphogenetic protein signaling cascade. Molecular and Cellular Biology, 25, 7711-7724. doi:10.1128/MCB.25.17.7711-7724.2005

[4] Kendall, S.E., Goldhawk, D.E., Kubu, C., Barker, P.A. and Verdi, J.M. (2002) Expression analysis of a novel p75(NTR) signaling protein, which regulates cell cycle progression and apoptosis. Mechanisms of Development, 117, 187-200. doi:10.1016/S0925-4773(02)00204-6

[5] Rochira, J.A., Cowling, R.A., Himmelfarb, J.S., Adams, T.L. and Verdi, J.M. (2010) Mapping of NRAGE domains reveals clues to cell viability in BMP signaling. Apoptosis, 15, 63-70. doi:10.1007/s10495-009-0427-6

[6] Matluk, N., Rochira, J.A., Karaczyn, A., Adams, T. and Verdi, J.M. (2010) A role for NRAGE in NF-kappaB activation through the non-canonical BMP pathway. BMC Biology, 8, 7. doi:10.1186/1741-7007-8-7

[7] Hudson, J.D., Shoaibi, M.A., Maestro, R., Carnero, A., Hannon, G.J. and Beach, D.H. (1999) A proinflammatory cytokine inhibits p53 tumor suppressor activity. The Journal of Experimental Medicine, 190, 1375-1382. doi:10.1084/jem.190.10.1375 
[8] Munaut, C., Boniver, J., Foidart, J.M. and Deprez, M. (2002) Macrophage migration inhibitory factor (MIF) expression in human glioblastomas correlates with vascular endothelial growth factor (VEGF) expression. $\mathrm{Neu}$ ropathology and Applied Neurobiology, 28, 452-460. doi:10.1046/j.1365-2990.2002.00416.x

[9] Petrenko, O., Fingerle-Rowson, G., Peng, T., Mitchell, R.A. and Metz, C.N. (2003) Macrophage migration inhibitory factor deficiency is associated with altered cell growth and reduced susceptibility to Ras-mediated transformation. The Journal of Biological Chemistry, 278, 11078-11085. doi:10.1074/jbc.M211985200

[10] Petrenko, O. and Moll, U.M. (2005) Macrophage migration inhibitory factor MIF interferes with the Rb-E2F pathway. Molecular Cell, 17, 225-236. doi:10.1016/j.molcel.2004.11.052

[11] Ito, K., Yoshiura, Y., Ototake, M. and Nakanishi, T. (2008) Macrophage migration inhibitory factor (MIF) is essential for development of zebrafish, Danio rerio. Developmental \& Comparative Immunology, 32, 664-672.

[12] Ito, T., Ito, N., Saatoff, M., Hashizume, H., Fukamizu, H., Nickoloff, B.J., Takigawa, M. and Paus, R. (2008) Maintenance of hair follicle immune privilege is linked to prevention of NK cell attack. Journal of Investigative Dermatology, 128, 1196-1206. doi:10.1038/sj.jid.5701183

[13] Suzuki, M., Takamura, Y., Maeno, M., Tochinai, S., Iyaguchi, D., Tanaka, I., Nishihira, J. and Ishibashi, T. (2004) Xenopus laevis macrophage migration inhibitory factor is essential for axis formation and neural development. The Journal of Biological Chemistry, 279, 2140621414. doi:10.1074/jbc.M311416200

[14] Rochira, J.A., Matluk, N.N., Adams, T.L., Karaczyn, A.A., Oxburgh, L., Hess, S.T. and Verdi, J.M. (2011) A small peptide modeled after the NRAGE repeat domain inhibits XIAP-TAB1-TAK1 signaling for NF-kappaB activation and apoptosis in P19 cells. PLoS One, 6, e20659. doi:10.1371/journal.pone.0020659

[15] Slack, R.S., Skerjanc, I.S., Lach, B., Craig, J. and Jardine, K. (1995) McBurney MW: Cells differentiating into neuroectoderm undergo apoptosis in the absence of functional retinoblastoma family proteins. The Journal of Cell Biology, 129, 779-788. doi:10.1083/jcb.129.3.779

[16] Tsukane, M., Yoshizaki, C. and Yamauchi, T. (2007) Development and specific induction of apoptosis of cultured cell models overexpressing human tau during neural differentiation: Implication in Alzheimer's disease. Analytical Biochemistry, 360, 114-122. doi:10.1016/j.ab.2006.10.003

[17] Suzuki, H., Nishihira, J., Koyama, Y. and Kanagawa, H. (1996) The role of macrophage migration inhibitory factor in pregnancy and development of murine embryos. Biochemistry \& Molecular Biology International, 38, 409416.

[18] Glozak, M.A. and Rogers, M.B. (1998) BMP4- and RAinduced apoptosis is mediated through the activation of retinoic acid receptor alpha and gamma in P19 embryonal carcinoma cells. Experimental Cell Research, 242, 165173. doi:10.1006/excr.1998.4075
[19] Nemajerova, A., Moll, U.M., Petrenko, O. and FingerleRowson, G. (2007) Macrophage migration inhibitory factor coordinates DNA damage response with the proteasomal control of the cell cycle. Cell Cycle, 6, 1030-1034. doi:10.4161/cc.6.9.4163

[20] Klingensmith, J., Matsui, M., Yang, Y.P. and Anderson, R.M. (2010) Roles of bone morphogenetic protein signaling and its antagonism in holoprosencephaly. American Journal of Medical Genetics. Part C, Seminars in Medical Genetics, 154C, 43-51. doi:10.1002/ajmg.c.30256

[21] Koga, K., Kenessey, A., Powell, S.R., Sison, C.P., Miller, E.J. and Ojamaa, K. (2011) Macrophage migration inhibitory factor provides cardioprotection during ischemia/reperfusion by reducing oxidative stress. Antioxidants \& Redox Signaling, 14, 1191-1202.

[22] Dennery, P.A. (2007) Effects of oxidative stress on embryonic development. Birth Defects Research Part C: Embryo Today, 81, 155-162. doi:10.1002/bdrc.20098

[23] Kobayashi-Miura, M., Nakamura, H., Yodoi, J. and Shiota, K. (2002) Thioredoxin, an anti-oxidant protein, protects mouse embryos from oxidative stress-induced developmental anomalies. Free Radical Research, 36, 949-956. doi:10.1080/1071576021000006626

[24] Kleemann, R., Kapurniotu, A., Mischke, R., Held, J. and Bernhagen, J. (1999) Characterization of catalytic centre mutants of macrophage migration inhibitory factor (MIF) and comparison to Cys81Ser MIF. European Journal of Biochemistry, 261, 753-766. doi:10.1046/j.1432-1327.1999.00327.x

[25] Kudrin, A. and Ray, D. (2008) Cunning factor: Macrophage migration inhibitory factor as a redox-regulated target. Immunology \& Cell Biology, 86, 232-238. doi:10.1038/sj.icb.7100133

[26] Takahashi, A., Iwabuchi, K., Suzuki, M., Ogasawara, K., Nishihira, J. and Onoe, K. (1999) Antisense macrophage migration inhibitory factor (MIF) prevents anti-IgM mediated growth arrest and apoptosis of a murine $\mathrm{B}$ cell line by regulating cell cycle progression. Microbiology and Immunology, 43, 61-67.

[27] Kobayashi, S., Satomura, K., Levsky, J.M., Sreenath, T., Wistow, G.J., Semba, I., Shum, L., Slavkin, H.C. and Kulkarni, A.B. (1999) Expression pattern of macrophage migration inhibitory factor during embryogenesis. Mechanisms of Development, 84, 153-156. doi:10.1016/S0925-4773(99)00057-X

[28] Ra, H., Kim, H.L., Lee, H.W. and Kim, Y.H. (2009) Essential role of p53 in TPEN-induced neuronal apoptosis. FEBS Letters, 583, 1516-1520. doi:10.1016/j.febslet.2009.04.008

[29] Hristoskova, S., Holzgreve, W., Zhong, X.Y. and Hahn, S. (2006) Macrophage migration inhibition factor is elevated in pregnancy, but not to a greater extent in preeclampsia. Archives of Gynecology and Obstetrics, 274, 25-28. doi:10.1007/s00404-005-0109-8

[30] Ietta, F., Todros, T., Ticconi, C., Piccoli, E., Zicari, A, Piccione, E. and Paulesu, L. (2002) Macrophage migration inhibitory factor in human pregnancy and labor. American Journal of Reproductive Immunology, 48, 404- 
409.

[31] Vigano, P., Cintorino, M., Schatz, F., Lockwood, C.J. and Arcuri, F. (2007) The role of macrophage migration inhibitory factor in maintaining the immune privilege at the fetal-maternal interface. Seminars in Immunopathology, 29, 135-150. doi:10.1034/j.1600-0897.2002.01152.x
[32] Faria, M.R., Hoshida, M.S., Ferro, E.A., Ietta, F., Paulesu, L. and Bevilacqua, E. (2010) Spatiotemporal patterns of macrophage migration inhibitory factor (Mif) expression in the mouse placenta. Reproductive Biology and Endocrinology, 8, 95. doi:10.1007/s00281-007-0074-3

\section{ABBREVIATIONS}

MIF: Macrophage migration inhibitory factor.

BMP: Bone morphogenic protein. 